\title{
Does salmon calcitonin cause cancer? A review and meta-analysis
}

\author{
G. Wells ${ }^{1}$ - J. Chernoff ${ }^{2}$ J. P. Gilligan ${ }^{3}$ - D. S. Krause ${ }^{3}$ (I)
}

Received: 27 July 2015 / Accepted: 24 September 2015 / Published online: 5 October 2015

(C) The Author(s) 2015. This article is published with open access at Springerlink.com

\begin{abstract}
Recently an association between the use of calcitonin and cancer has been postulated. We reviewed the biological rationale and performed an additional analysis of historical data with respect to the possibility. An association cannot be excluded, but the relationship is weak and causality is unlikely. The purpose of the present study is to review the strength of association and likelihood of a causal relationship between use of calcitonin and cancer. We reviewed the evidence for this association, including the molecular signaling mechanisms of calcitonin, preclinical data, an "experiment of nature," and the results of a previous meta-analysis which showed a weak association. We performed an additional meta-analysis to incorporate the data from a novel investigational oral formulation of salmon calcitonin. Review of the literature did not identify a cellular signaling mechanism of action which might account for a causal relationship or toxicologic or postmarketing data to support the thesis. Additional clinical results incorporated into previous meta-analyses weakened but did not completely negate the possibility of association. A causal association between calcitonin use and malignancy is unlikely, as there is little biological plausibility.
\end{abstract}

D. S. Krause

dkrause@tarsatherapeutics.com

1 University of Ottawa Heart Institute, Ottawa, Ontario, Canada K1Y 4W7

2 Fox Chase Cancer Institute, 333 Cottman Ave \# 307, Philadelphia, USA

3 Tarsa Therapeutics Inc, 8 Penn Center, 1628 JFK Blvd., Philadelphia, PA 19103, USA
The preponderance of nonclinical and clinical evidence also does not favor a causal relationship.

Keywords Cancer $\cdot$ Meta-analysis $\cdot$ Review $\cdot$ Salmon calcitonin

\section{Introduction}

Calcitonin is a 32-amino acid peptide hormone present in mammals, fish, birds, and reptiles. In humans, calcitonin $(\mathrm{hCT})$ is produced by the parafollicular $(\mathrm{C})$ cells of the thyroid gland in response to increases in extracellular levels of calcium. The exact role of calcitonin in human health and disease continues to be elucidated, but it has long been thought to play an important role in bone and mineral homeostasis, particularly with respect to its ability to regulate calcium metabolism. However, some questions remain about the exact physiologic role of calcitonin. For example, no calcitonin deficiency state has ever been described. This has led some to hypothesize that calcitonin is a vestigial hormone [1]. However, recently, more complex signaling pathways of calcitonin in bone physiology have been described, and as is the case with parathyroid hormone (PTH), the pharmacologic activity may differ from the physiologic role [2].

Calcitonin has long been known to have an anti-resorptive effect on bone, mediated via calcitonin receptors $(\mathrm{CTr})$ present on osteoclasts. $\mathrm{CTr}$ are also present in other tissues, most notably kidney and brain; in situ hybridization studies suggest a broader spectrum of tissues containing $\mathrm{C} T r$, including prostate, as well as the exocrine glands in the pancreas, and stomach [3]. Salmon calcitonin (sCT) has approximately $50 \%$ amino acid homology to hCT but has anti-resorptive properties in human bone approximately 40 times as potent as those of hCT and a longer half-life [4]. It is these properties of sCT 
that have allowed its use for decades as a therapeutic agent for postmenopausal osteoporosis, Paget's disease, and other diseases of bone. sCT was first licensed for use in the USA in 1975. The most commonly used form of sCT, nasal spray, has been used in the USA for approximately 20 years and is currently indicated for the treatment of osteoporosis in women greater than 5 years postmenopause when alternative treatments are not suitable [5].

In its decades of use, few safety concerns have been raised for calcitonin. However, in 2012, the European Medicines Agency (EMA) suspended calcitonin nasal spray from the market and limited the duration of use of other calcitonin products due to a putative association with cancer [6]. This was triggered by the observation of a greater number of prostate cancer cases at the time of an interim analysis of an oral investigational $\mathrm{SCT}$ product being studied in osteoarthritis. The product in question, SMC021, was being evaluated in two large phase 3 trials of osteoarthritis (OA). Upon conclusion of the trials, no imbalance in prostate cancer was observed, and the issue was not discussed in the final publication of these trials [7, 8]. However, the interim findings prompted a retrospective review of prior randomized trials, which led to the EMA's action. In March 2013, the US Food and Drug Administration (FDA) convened a joint meeting of the Advisory Committee for Reproductive Health Drugs and the Drug Safety and Risk Management Advisory Committee to address this issue in the US. Subsequent to this meeting, in March 2014, the US prescribing information for all SCT products (nasal spray and injectable) was revised to note that the results of a meta-analysis "suggest(s) an increased risk of malignancies in calcitonin-salmon-treated patients compared to placebo" [5]. However, no "black box" warning was incorporated. Also, the indication was revised such that it is now indicated for treatment of postmenopausal osteoporosis in women greater than 5 years postmenopause "when alternative treatments are not suitable." The statement that "fracture reduction efficacy has not been demonstrated" was also added.

In this paper, we review the biological plausibility of the putative association of calcitonin and oncogenicity with respect to cellular signaling mechanisms, the preclinical carcinogenicity data, and results of an additional meta-analysis which incorporates the results of another investigational oral formulation of sCT. We also discuss the implications of an experiment of nature, medullary thyroid cancer.

\section{Signaling mechanisms}

As noted above, calcitonin elicits its biologic effect by binding to $\mathrm{CTr}$, which is a member of a subfamily of the seven transmembrane domain G-protein-coupled receptor superfamily that includes glucagon-like peptide 1, glucagon, pituitary adenylate cyclase (AC)-activating polypeptide, corticotropin- releasing factor, gastric inhibitory polypeptide receptors, and PTH/PTH-related peptide [9]. It has long been known that some malignant cells express $\mathrm{C} \operatorname{Tr}[10]$. Binding to $\mathrm{C} \operatorname{Tr}$ activates $A C$ which results in an increase of intracellular cyclic adenosine monophosphate (cAMP). Increases in cAMP directly activate two classes of effector proteins: protein kinase A (PKA) and the guanine nucleotide exchange factor EPAC. With respect to cancer-relevant phenomena, cAMP/PKA is known to regulate cell proliferation, apoptosis, and motility; in most cells, cAMP serves to inhibit cell growth [11]. The growth-suppressive activities of cAMP/PKA are mediated by a number of signaling pathways, and this effect of cAMP, mediated via $\mathrm{SCT}$, has been shown, e.g., in a line of malignant breast cancer cells [12]. The best understood link between PKA and suppression of proliferation occurs by repressing ERK. In addition, it should be noted that one of the best studied prostate cancer cell lines, DU-145, has been reported to show decreased ERK activity and decreased c-Jun expression in response to calcitonin [13]. Since ERK activity is closely correlated to cell cycle progression, it is doubtful that calcitonin would promote proliferative behavior in this particular prostate cancer cell line.

The Shah group has reported that (a) exogenously added $\mathrm{hCT}$ induces DNA synthesis in prostate cancer LNCaP and PC-3M cells [14], (b) hCT, acting through Gs/cAMP and $\mathrm{Ca} 2+$ pathways, induces production of a $\mathrm{CD} 44$ splice variant that is associated with increased invasiveness [15-17], (c) calcitonin stimulates angiogenesis [18], and (d) calcitonin can promote in vivo metastases of $\mathrm{PC} 3$ prostate cancer cells [19]. These data point to an oncogenic effect for calcitonin, via activation of AC and increases in CAMP, in these prostate cancer cell lines. These results, however, conflict with other published studies of the biology of calcitonin. For example, Macchia et al. reported that elevated cAMP inhibits the growth of LNCaP cells and the Ritchie group demonstrated that hCT decreased proliferation of both PC-3 and DU-145 cells, consistent with the role of elevated cAMP suppressing cell growth $[20,21]$. These results are further supported by Segawa, who reported that DU-145 cells treated with calcitonin resulted in decreased ERK activity and decreased c-Jun [22]. Since ERK activity is closely correlated with suppression of cell proliferation, these results support the findings of Ritchie with respect to the results for PC-3 and DU-145 cell lines. Nakamura reported that gastrin-releasing peptide and calcitonin gene-related peptide (CGRP) increased tumor cell invasion of DU-145 cells whereas calcitonin did not [23]. GRP and CGRP also increased the tumor invasiveness of PC-3 cells while calcitonin did not. The effect of CGRP is of interest since the exposure required for calcitonin to achieve a positive effect on $\mathrm{LNCaP}$ cells was $10 \mathrm{ng} / \mathrm{ml}$, approximately 1000 times greater than the Cmax achieved with nasal sCT [21]. These highly elevated levels of calcitonin have the potential to cross react with CGRP receptors and might explain 
the some of the contradictory findings of Shah et al., calling into question his postulate linking calcitonin to prostate cancer.

More recently, Tsagaraki et al. conducted an experiment in which MG63 osteosarcoma cells exposed to calcitonin demonstrated an NF-kB-dependent induction of fibronectin, an extracellular matrix component, and matrix metalloproteinase-9 (MMP-9), a combination that is often associated with oncogenic processes [24]. However, in response to calcitonin, these same cells were also found to express elevated levels of tissue inhibitor of metalloprotease (TIMP)1 and TIMP-2, which oppose the action of MMP-9 and MMP2 , respectively. Given these complexities, and the fact that these studies were carried out in vitro in a single cell line, their clinical relevance is not clear.

In summary, there are no data which demonstrate that calcitonin can induce carcinogenic transformation. The data with respect to calcitonin's ability to promote tumor aggressiveness are inconsistent and contradictory.

\section{Preclinical studies}

Agents for use as human pharmaceuticals are required to undergo rigorous in vitro and in vivo testing for general toxicity, genotoxicity, and carcinogenicity prior to licensure. The latter is typically performed in multiple species. In such studies, SCT was not mutagenic in multiple bacterial strains, nor was it clastogenic in chromosome aberration tests of Chinese hamster ovary V79 cells [5]. Neither 1-year chronic toxicity studies in Sprague-Dawley rats nor formal carcinogenicity studies of sCT in Sprague-Dawley rats and CD-1 mice dosed for up to 2 years at doses 39 to 390 times the maximum recommended subcutaneous nor intranasal doses yielded evidence of carcinogenicity. The sole issue arising in nonclinical testing was the finding of decreased latency period in nonmalignant pituitary adenomas in rodents, which occur with a background frequency of 34-94\% [7]. However, there was no evidence of malignant transformation of these tumors, nor is it believed to be relevant to human use. The prescribing information for current calcitonin products states "In female Sprague Dawley rats, the incidence of pituitary adenomas after two years was high in all treatment groups (between $80 \%$ and $92 \%$ including the control groups) such that a treatment-related effect could not be distinguished from natural background incidence" [5].

Consistent with these data, Sondergaard et al. created transgenic mice over-expressing sCT. These mice were not noted to have an increased risk of cancer, although the publication did not state that toxicologic examination was performed [25].

In summary, in vivo animal data did not identify a risk of malignancy following exposure to $\mathrm{sCT}$.

\section{Calcitonin-producing tumor}

If calcitonin does not itself cause cancer, a possible alternative hypothesis to support a putative relationship between calcitonin and increased cancer risk is to suggest that calcitonin elicits a nonspecific tumor-promoting effect. An experiment of nature is medullary thyroid cancer (MTC), an unusual but not rare tumor of the parafollicular (C) cells of the thyroid. It may occur sporadically or as a component of several multiple endocrine neoplasia (MEN) constellations. Individuals with MTC may elaborate extraordinarily high levels of hCT, and serum $\mathrm{hCT}$ is used as both a diagnostic and prognostic factor in the approach to treatment [26]. If the hypothesis that calcitonin is a nonspecific oncogen is correct, it could be expected that individuals with MTC would have a high likelihood of second primary cancers, as many tumor types are associated with bidirectional risk of a second primary malignancy. The US Surveillance, Epidemiology and End Results (SEER) program collects data on all persons diagnosed with cancer in specific geographic regions. Using nine SEER registries, Ronckers et al. examined the likelihood of a second primary malignancy after the occurrence of thyroid cancer(s) [27]. The authors were able to identify 884 cases of MTC which were followed for a median of 8 years. These individuals did not show evidence of an increased risk of secondary malignancies, as opposed to individuals with papillary and follicular thyroid carcinoma, who did show evidence of increased risk. These data do not support an association between hCT and increased risk of malignancy.

\section{Meta-analysis}

In November 2010, the sponsor of an investigational oral calcitonin product being studied in two phase 3 trials (each 2year duration) of OA informed regulatory authorities of an imbalance in prostate cancer in male study participants [6, 7]. At the time of the notification, one of the trials was ongoing and the other complete. Following an extensive investigation, including retrospective screening of study participants and measurement of serum prostate-specific antigen (PSA) levels and completion of the trials, final data did not ultimately reveal an increased risk of prostate cancer $(5.4 \%$ [20/365] of male calcitonin recipients vs. $4.0 \%$ [16/405] of placebo recipients) [7]. In subjects diagnosed with prostate cancer, Gleason scores did not differ significantly for subjects who had received SCT compared to placebo subjects (6.9 vs. $6.4, p=$ 0.27). Most of these individuals were found to have had high PSA levels at baseline which would have been discovered on routine screening. PSA levels were not accelerated in $\mathrm{SCT}$ recipients compared to placebo.

Following the initial signal of a potential increase in prostate cancer incidence, a literature search was conducted, and 
the sponsor, Novartis Pharmaceuticals, conducted a metaanalysis of 17 historical studies of nasal spray calcitonin [6, 28]. The methods and results from this analysis have been extensively reported in publically available regulatory documents $[6,7,29]$. All but two of these trials were limited to women. They included daily doses ranging from 100 to $400 \mathrm{IU}$ per day (the licensed dose of Miacalcin ${ }^{\circledR}$ nasal spray is $200 \mathrm{IU} /$ day). The results of this analysis suggested a small increased risk of developing any cancer in calcitonin recipients compared to placebo (odds ratio [OR] 1.61 [95\% confidence interval (CI), 1.11-2.34]). The authors then excluded four trials in which no cases of cancer occurred "according to the Peto method" [28]. In this analysis, the OR was 1.9 (CI 1.27-2.84). The most frequently reported cancer was basal cell carcinoma, but many different types of cancer were reported in calcitonin and placebo recipients. Analysis by dose of calcitonin did not suggest any dose response, and the duration of exposure was similar in calcitonin-treated cases to placebo-treated cases (CI 21.8 vs. 22.4 months). The increased risk observed in this analysis was almost entirely attributable to one large study, known as the PROOF trial $[29,30]$.

In 2013, the US FDA performed its own meta-analysis, incorporating data from Novartis' investigational oral calcitonin into the above-described nasal spray analysis (and one additional historical nasal spray study) [7]. These data were subsequently included in the prescribing information for all calcitonin products in the USA [5]. In this analysis, which was expressed in terms of risk difference (RD) rather than OR, the $\mathrm{RD}$ for the development of any cancer in SCT-treated patients exceeded that of placebo-treated patients by $1 \%(95 \% \mathrm{CI}$, $0.3,1.6)$. When basal cell cancer was excluded, the risk was not significant as the RD was $0.5 \%(95 \% \mathrm{CI},-0.1,1.2)$, despite which the prescribing information states that "imbalances in risk were still observed" [5].

An alternative formulation of recombinant $\mathrm{SCT}$ for oral administration is in late clinical development for postmenopausal osteoporosis [31]. Based upon publically available data and data from Tarsa Therapeutics' phase 2 and 3 studies of its investigational oral $\mathrm{sCT}$, one of the authors $(\mathrm{GW})$ performed an additional meta-analysis to further explore a possible association between $\mathrm{SCT}$ and cancer. The analysis included data from 22 randomized, controlled clinical trials with calcitoninsalmon (nasal spray or oral investigational oral formulations). Essentially, this analysis incorporated available data from the original 17 trial meta-analyses of Heep [28] (including the studies with zero events), the three large oral phase 3 trials of an investigational oral of calcitonin formulation studies sponsored by Novartis (including the two phase 3 osteoarthritis studies and a third study in postmenopausal women with osteoporosis), and the two trials of a different oral formulation of sCT sponsored by Tarsa Therapeutics, Inc (Fig. 1, study 201 and study 801). These two trials, each approximately 1 year in duration, have been described elsewhere; neither trial showed an increased incidence of cancer in calcitonin recipients, nor was there an excess risk when data from the two trials were pooled [31-33]. The analysis employed methods that were intended to replicate the data in Table 9 of the FDA's background document for the joint advisory committee meeting [7]. Therefore, this analysis considered RD estimates for each trial and a meta-analysis using a Mantel-Haenszel-type method to estimate the pooled RD for all strata, assuming a fixed effects model.

First, the data displayed in Table 9 of the FDA's briefing book were reproduced utilizing RevMan software version 5.2 (http://tech.cochrane.org/RevMan). This software employs the same fixed effect method and uses a forest plot to display the frequency data and RD estimates with $95 \%$ CIs (provided both in numeric and graphic formats). In addition, the weight of the contribution of each study to the overall, meta-analytic RD result is provided. The standard tests for association as well as the Cochran Q-test and $I^{2}$ measure for heterogeneity were calculated. The data derived by the US FDA were replicated exactly, except for slight differences in the $95 \%$ CI values for zero-event studies: the overall RD was identical (please note that there is an apparent addition error in the denominator for the placebo group in Table 9 of the FDA's background document, which should total 4706 and not 4687). Secondly, the adverse events consistent with cancer were incorporated from the two Tarsa-sponsored studies. The resultant meta-analysis and forest plot are displayed in Fig. 1. A total of 11,489 unique individuals, including males, were included in this analysis. The trials ranged in duration from 6 months to 5 years; the two Tarsa-sponsored studies were 48 weeks and 1 year in duration $[31,32]$.

The forest plot displays the frequency data and RD estimates with $95 \%$ CIs (provided in both numeric and graphic formats) for each of the studies. The Cochrane Q-test ( $p=$ 0.44 ) and the $I^{2}$ measure indicate that heterogeneity is not an issue. As a result, weights based on the fixed effects model were used to combine the study estimates, yielding the metaanalytic RD of $0.90 \%(95 \% \mathrm{CI}, 0.21$ to $1.58 \%)$ and the test of association indicates that this RD is significant $(p=0.01)$. As noted previously, the data are heavily influenced by a single large 5-year trial (CT320 [PROOF]), resulting in a slight increased risk of any malignancy [30].

\section{Postmarketing data}

Data derived from spontaneous reports of adverse events to regulatory authorities have well-known limitations including absence of proof of causality, the presence of co-morbidities, and other limitations such as knowledge of duration of use and total exposure. Nonetheless, $\mathrm{sCT}$ has been available and widely used for many decades, such that even a faint signal might be apparent. In anticipation of the 2013 advisory committee, the 
Fig. 1 Meta-analysis of all available nasal and oral trials

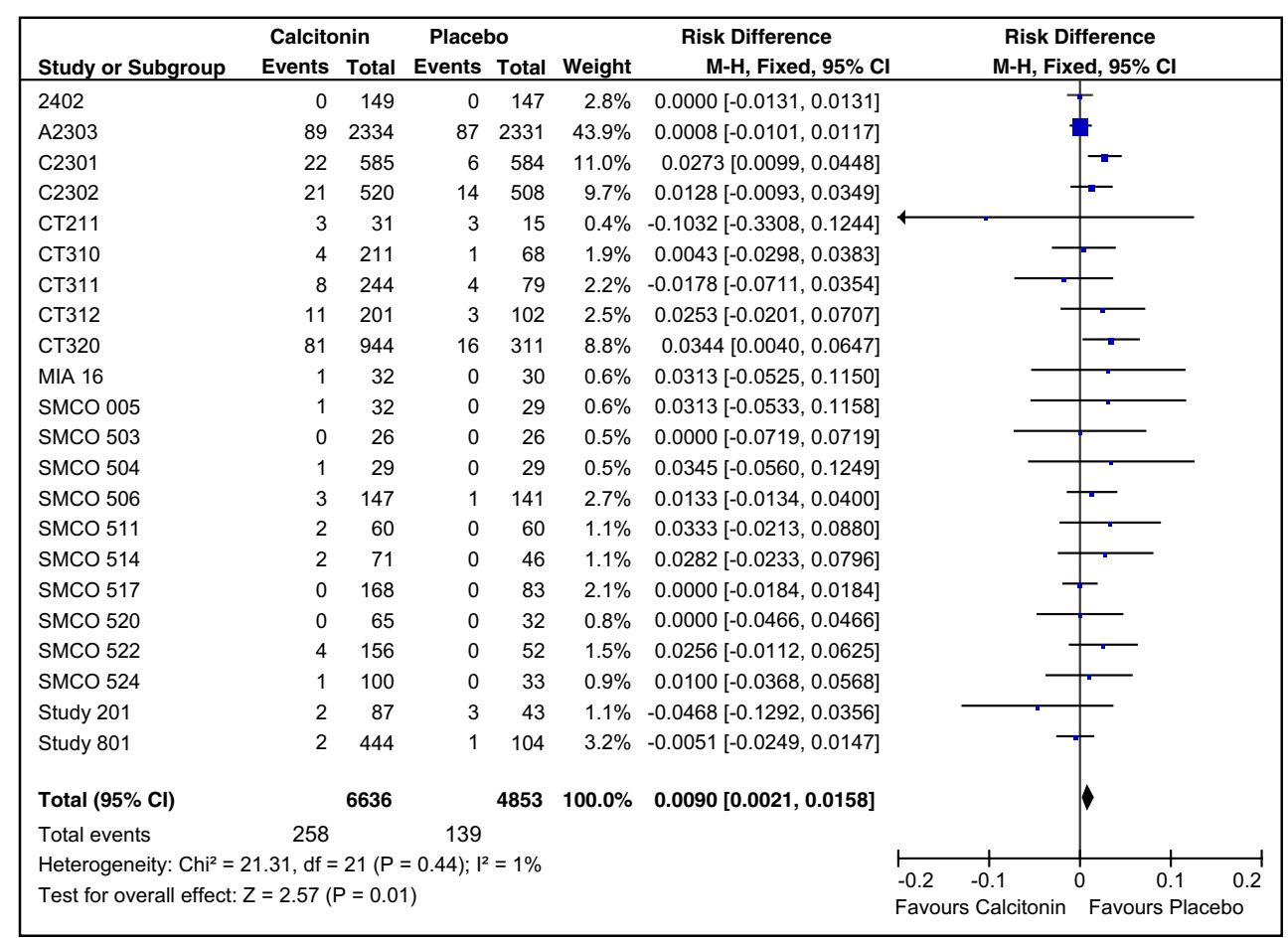

US FDA performed a review of spontaneously reported adverse events of malignancy in calcitonin recipients and a data mining exercise to look at a possible association [7]. Usage data included reports from the US Adverse Event Reporting System (AERS) and multiple proprietary drug utilization databases. The agency concluded that its findings "did not identify any potential signal for prostate cancer or other malignancies in the postmarketing data," nor in the data mining exercise [7].

\section{Discussion}

Calcitonin has been in use for decades, and many millions of individuals have been exposed to pharmaceutical doses of sCT; it remains in use in many countries throughout the world, including the USA. An association with cancer, particularly a causal relationship, would clearly be important information.

Our meta-analysis is consistent with a small increased risk of cancer in calcitonin recipients when combining all types of malignancies; however, the data are not strongly persuasive of such an association in our opinion; the evidence of a causal relationship is even weaker. This interpretation is consistent with others who have also found the evidence to be of poor quality [34]. A large nested case-control study of the use of calcitonin nasal spray in women with postmenopausal osteoporosis found that there was no association with "all cancer" but that there was a positive association (increased risk) for liver cancer and a negative association (decreased risk) for breast cancer in calcitonin users [35]. In our opinion, these results are likely due to chance given the many types of cancer for which a possible association was sought. The authors concluded that that the results "did not completely support" the decision to discontinue use of calcitonin.

For many years, the criteria proposed by Bradford Hill in 1965 have been used to differentiate a cause and effect relationship from a noncausal association with respect to epidemiologic and environmental factors and disease [36]. The same criteria have been useful in the pharmacovigilance arena [37]. These criteria include strength, consistency, specificity, temporality, biological gradient, plausibility, coherence, and analogy.

With respect to the strength of association, the signal is weak and not statistically significant for any individual study. The lower bound of the $95 \% \mathrm{CI}$ of the RD is close to 0 , and specificity and biological plausibility are absent.

The data are inconsistent on a number of levels, including results of formal preclinical toxicology data, in which calcitonin administration resulted in a lack of tumors in tissues of rodent species/strains which have a background incidence of spontaneous tumors. Therefore, the postulate of nonspecific tumor promotion is inconsistent with basic experimental carcinogenicity findings. Furthermore, no signal was noted in the largest study (Fig. 1, A2303) with the greatest patient-years of exposure. No temporality was observed; i.e., time to onset was not different in the intranasal group compared to placebo recipients. No signal is apparent in postmarketing surveillance data despite many years of use.

Specificity is entirely absent; the original NVS metaanalysis of 17 intranasal trials included 19 different types of tumors. The concept of nonspecific, multisite tumor 
promoters is inconsistent with the fundamental principle that tumor promoters are organ specific, based on several decades of experimental carcinogenesis studies elucidating the mechanism of tumor formation.

A biological gradient is not apparent, as no dose-response was evident in the FDA's analysis [7]. In PROOF, there was an inverse relationship between total exposure and development of the two most commonly reported malignancies, BCC and breast cancer.

There are no experimental data that show a cause and effect relationship, aside from prostate cancer data from the Shah lab. Ironically, prostate cancer is the only cancer for which a systematic evaluation was performed in the clinic, in two large OA trials. For this tumor type, the thesis of increased risk was ultimately refuted.

We are not aware of an analogy, e.g., in which a drug substance serves as a nonspecific oncogen or tumor promoter and whose effects are not observed in formal carcinogenicity studies.

None of these factors alone can disprove a causal relationship, but together, they provide a compelling argument against causality. Based on the preclinical literature, the known mechanism of action of calcitonin, and the receptor distribution, there is no real biological plausibility for a "pan cancer" causal association, nor for any single cancer type.

Our analysis, like those preceding it, has important limitations. None of the 22 trials included in the meta-analysis was designed specifically to identify an increased risk of malignancy, nor were cancer cases adjudicated in blinded fashion. In all cases, the analyses refer to adverse events reported as malignancy or consistent with a malignant adverse event. Furthermore, the enrolment criteria and duration of use varied in these trials. The exposure to $\mathrm{SCT}$ afforded by the various routes of administration and investigational oral formulations may not be comparable.

\section{Conclusion}

There is a need for additional agents for the treatment of osteoporosis, and all currently available agents have limitations. Calcitonin has been shown to have a modest but consistent salutary effect on lumbar spine bone mineral density, and at a dose of $200 \mathrm{IU} /$ day, intranasal calcitonin was shown to have a positive effect on the risk of vertebral fracture [30, 31]. At present, the therapeutic milieu for the treatment of postmenopausal osteoporosis is not optimal, as the use of available agents has contracted, likely due to fears on the part of patients and providers regarding the complications of anti-resorptive agents, and recommendations for "drug holidays" [38, 39]. To our knowledge, calcitonin has never been associated with atypical femoral fracture or osteonecrosis of the jaw. Calcitonin may have a role in patients for whom other agents are unsuitable or otherwise contraindicated, consistent with its current US prescribing information. An oral formulation, currently in development, may be desirable for some patients.

Current understanding of the biology and mechanism of action of SCT does not support a role as an oncogen or tumor-accelerating agent. Such evidence is lacking in preclinical carcinogenicity, genotoxicity, and mutagenicity studies and, in general, biological plausibility. Consideration of all of the available data suggests that the association between calcitonin and cancer is weak, antithetical to the known biology of calcitonin, and not causal.

Acknowledgments The authors thank Neil Binkley, MD, for thoughtful critiques of earlier versions of this paper.

Funding This work was funded by Tarsa Therapeutics Inc. The data analysis was performed by GW based upon publically available information and data supplied by Tarsa Therapeutics. All authors had access to the data and made substantial contributions to the manuscript. The original manuscript was drafted by DK. All authors reviewed and approved the final version to be published.

\section{Compliance with ethical standards}

Conflicts of interest GW and JC received consulting fees from Tarsa Therapeutics. DK and JG are employees and shareholders of Tarsa Therapeutics.

Ethical approvals This work does not contain original studies with human participants performed by any of the authors, with the exception of previously published studies which were performed in accordance with the ethical standards of the Declaration of Helsinki [29-31].

Open Access This article is distributed under the terms of the Creative Commons Attribution-NonCommercial 4.0 International License (http:// creativecommons.org/licenses/by-nc/4.0/), which permits any noncommercial use, distribution, and reproduction in any medium, provided you give appropriate credit to the original author(s) and the source, provide a link to the Creative Commons license, and indicate if changes were made.

\section{References}

1. Hirsch PF, Baruch H (2003) Is calcitonin an important physiologic substance? Endocrine 21:201-08

2. Martin TJ, Sims NA (2015) Calcitonin physiology, saved by a lysophospholipid. J Bone Miner Res 30:212-15

3. Mori I, Ishii A, Nakamura M et al (2006) Expression and cellular localization of calcitonin receptor: RT-PCR and in situ hybridization studies. Cell Mol Biol (Noisy-le-grand) 52:9-13

4. Niall HD, Keutmann HT, Copp DH, Potts JT (1969) Amino acid sequence of salmon ultimobranchial calcitonin. Proc Natl Acad Sci U S A 64:771-8

5. Miacalcin ${ }^{\circledR}$ nasal spray [package insert]. Novartis Pharmaceuticals Corp. East Hanover, New Jersey 07936; March 2014

6. CHMP Referral Assessment Report Procedure number: EMEA/H/ A-31/1291 24 July 2012 
7. U.S. Food and Drug Administration. Background document for meeting of Advisory Committee for reproductive health drugs and drug safety and risk management advisory committee. March 5, 2013. Available at: http://www.fda.gov/downloads/ AdvisoryCommittees/CommitteesMeetingMaterials/Drugs/ ReproductiveHealthDrugsAdvisoryCommittee/UCM341779.pdf

8. Karsdal MA, Byrjalsen I, Alexandersen P et al (2015) Treatment of symptomatic knee osteoarthritis with oral salmon calcitonin: results from two phase 3 trials. Osteoarthr Cart 23:532-43

9. Hay D, Poyner DR, Quirion R (2008) Status of the calcitonin generelated peptide subtype 2 receptor. Pharmacol Rev 60:143-145

10. Findlay D, Sexteon PM (2004) Calcitonin. Growth Factors 22:21724

11. Houslay MD, Kolch W (2000) Cell-type specific integration of cross-talk between extracellular signal-regulated kinase and cAMP signaling. Mol Pharmacol 58:659-68

12. Ng KW, Livesey SA, Larkins RG, Martin TJ (1983) Calcitonin effects on growth and on selective activation of type II isoenzyme of cyclic adenosine 3'5'-monophosphate-dependent protein kinase in T47D human breast cancer cells. Cancer Res 43:794-800

13. Blok LJ, de Ruiter PE, Brinkmann AO (1998) Forskolin-induced dephosphorylation of the androgen receptor impairs ligand binding. Biochemistry 37:3850-57

14. Chien J et al (2001) Calcitonin is a prostate epithelium-derived growth stimulatory peptide. Mol Cell Endocrinol 181:69-79

15. Iczkowski KA, Omara-Opyene AL, Kulkarni TR, Pansara M, Shah GV (2005) Paracrine calcitonin in prostate cancer is linked to CD44 variant expression and invasion. Anticancer Res 25:2075-2083

16. Sabbisetti VS et al (2005) Calcitonin increases invasiveness of prostate cancer cells: role for cyclic AMP-dependent protein kinase A in calcitonin action. Int J Cancer 117:551-560

17. Shah GV et al (1994) Calcitonin stimulates growth of human prostate cancer cells through receptor-mediated increase in cyclic adenosine 3',5'-monophosphates and cytoplasmic $\mathrm{Ca} 2+$ transients. Endocrinology 134:596-602

18. Chigurupati S, Kulkarni T, Thomas S, Shah G (2005) Calcitonin stimulates multiple stages of angiogenesis by directly acting on endothelial cells. Cancer Res 65:8519-29

19. Shah GV et al (2008) Calcitonin promotes in vivo metastasis of prostate cancer cells by altering cell signaling, adhesion, and inflammatory pathways. Endocr Relat Cancer 15:953-64

20. Macchia V, Di Carlo A, De Luca C, Mariano A (2001) Effects of cyclic adenosine-monophosphate on growth and PSA secretion of human prostate cancer cell line. Int J Oncol 18:1071-76

21. Ritchie CK, Thomas KG, Andrews LR, Tindall DJ, Fitzpatrick LA (1997) Effects of the calciotrophic peptides calcitonin and parathyroid hormone on prostate cancer growth and chemotaxis. Prostate 30:183-187

22. Segawa N et al (2001) Phosphorylation of mitogen-activated protein kinase is inhibited by calcitonin in DU145 prostate cancer cells. Cancer Res 61:6060-63

23. Nakamura M, Han B, Nishishita T, Bai Y, Kakudo K (2007) Calcitonin targets extracellular signal regulated kinase signaling pathway in human cancers. J Mol Endocrinol 39:375-384
24. Tsagaraki I, Phenkos C, Tsilibary E et al (2013) Calcitonin-induced NF-KB activation up-regulates fibronectin expression in MG63 osteosarcoma cells. Anticancer Res 33:4901-06

25. Sondergaard BC, Catala-Lehnen HAK et al (2012) Mice overexpressing salmon calcitonin have strongly attenuated osteoarthritic histopathological changes after destabilization of the medial meniscus. Osteoarthr Cartil 20:136-43

26. Kloos RT, Eng C, Evans DB et al (2009) Medullary thyroid cancer: management guidelines of the American Thyroid Association. Thyroid 19:565-612

27. Ronckers CM, McCarron P, Ron E (2005) Thyroid carcinoma and multiple tumors in the SEER cancer registries. Int J Cancer 117: 281-88

28. Heep $\mathrm{M}$ et al (2012) Calcitonin use and risk of malignancy: a metaanalysis of 17 RCTs in patients with osteoporosis. Presented at the American Society of Bone and Mineral Research Annual Meeting 2012. Abstract 1234, October 15, 2012

29. Novartis Pharmaceuticals. Briefing book prepared for the joint meeting of the Advisory Committee for Reproductive Health Drugs and the Drug Safety and Risk Management Advisory Committee. 2013 Accessed at: http://www.fda.gov/downloads/ AdvisoryCommittees/CommitteesMeetingMaterials/Drugs/ ReproductiveHealthDrugsAdvisoryCommittee/UCM341781.pdf

30. Chesnut CH, Silverman S, Andriano K et al (2000) A randomized trial of nasal spray salmon calcitonin in postmenopausal women with established osteoporosis: the prevent recurrence of osteoporotic fracture study. Am J Med 109:267-76

31. Binkley N, Bolognese M, Sidorowicz-Bialynicka A et al (2012) A phase 3 trial of the efficacy and safety of oral recombinant calcitonin: the oral calcitonin in postmenopausal osteoporosis (ORACAL) trial. J Bone Miner Res 27:1821-29

32. Binkley N, Bone H, Gilligan JP, Krause D (2014) Efficacy and safety of oral recombinant salmon calcitonin tablets in postmenopausal women with low bone mass and increased fracture risk: a randomized, placebo-controlled trial. Osteoporos Int 25:2649-56

33. Krause D, Hernandez NAS, Vitagliano M, et al (2012) One year use of oral recombinant salmon calcitonin is not associated with increased risk of cancer. Presented at the American Society of Bone and Mineral Research Annual Meeting 2013. Presentation Number LB-MO17

34. Overman RA, Borse M, Gourlay ML (2013) Salmon calcitonin use and associated cancer risk. Ann Pharmacother 47:1675-84

35. Sun L, Lin M, Muo C et al (2014) Calcitonin nasal spray and increased cancer risk: a population based nested case-control study. J Clin Endocrinol Metab 99:4259-64

36. Hill AB (1965) The environment and disease: association or causation? Proc R Soc Med 58:295-300

37. Edwards I (2012) Considerations on causality in pharmacovigilance. Int J Risk Saf Med 24:41-54

38. Wysowski DK, Greene P (2013) Trends in osteoporosis treatment with oral and intravenous bisphosphonates in the United States, 2002-2012. Bone 57(2):423-428

39. Black D, Bauer D, Schwartz A, Cummings S, Rosen C (2012) Continuing bisphosphonate treatment for osteoporosis-for whom and for how long? N Engl J Med 366(22):2051-3 